\title{
*-PURE SUBGROUPS OF COMPLETELY DECOMPOSABLE ABELIAN GROUPS
}

\author{
LOYISO G. NONGXA
}

\begin{abstract}
In this note we prove that
(i) homogeneous *-pure subgroups of completely decomposable groups are completely decomposable,

(ii) *-pure subgroups of finite rank completely decomposable groups are almost completely decomposable.

We also characterize those reduced completely decomposable groups $G$, with $T(G)$ satisfying the maximum condition, any *-pure subgroup of which is also completely decomposable.
\end{abstract}

In [2], a subgroup $H$ of a torsion-free group $G$ is said to be $*$-balanced if $H$ is balanced in $G$ and

$$
\left\langle H^{*}(\tau)\right\rangle_{*}=H \cap\left\langle G^{*}(\tau)\right\rangle_{*}
$$

for every type $\tau$. We shall say that $H$ is *-pure in $G$ if $H$ is pure in $G$ and equation (i) above is satisfied for every type $\tau$.

Throughout this note, unless otherwise specified; $G$ will denote a completely decomposable abelian group and its extractable typeset will be denoted by $\mathcal{E}(G)$. We let $G=\bigoplus_{\tau \in \mathcal{E}(G)} G_{\tau}$ be a homogeneous decomposition of $G$ and, for every $\tau \in \mathcal{E}(G), \pi_{\tau}: G \rightarrow G_{\tau}$ is the projection such that ker $\pi_{\tau}=\bigoplus_{\tau^{\prime} \neq \tau_{\tau}} G_{\tau^{\prime}}$.

In [4] we proved that homogeneous pure subgroups of $G$ are also completely decomposable provided that $\mathcal{E}(G)$ is countable. The following theorem implies that the countability condition of $\mathcal{E}(G)$ can be relaxed if we consider homogeneous *-pure subgroups of $G$.

THEOREM 1. Homogeneous *-pure subgroups of completely decomposable groups are completely decomposable.

PROOF. Let $H$ be a homogeneous *-pure subgroup of a completely decomposable group $G$. Let $0 \neq h \in H$ and suppose that type ${ }_{G}(h)=\tau_{0}$. Then $h=\sum_{\tau \geq \tau_{0}} \pi_{\tau}(h)$. If $\tau_{0} \notin \mathcal{E}(G)$, then

$$
h=\sum_{\tau>\tau_{0}} \pi_{\tau}(h) \in H \cap G^{*}\left(\tau_{0}\right)=\left\langle H^{*}\left(\tau_{0}\right)\right\rangle_{*}=\{0\},
$$

a contradiction. Thus $\tau_{0} \in \mathcal{E}(G)$ and $\pi_{\tau_{0}}(h) \neq 0$. Hence $H \cong \pi_{\tau_{0}}(H) \subseteq G_{\tau_{0}}$. Since $\pi_{\tau_{0}}(H)$ is homogeneous of type $\tau_{0}$ and is a subgroup of a homogeneous completely decomposable group $G_{\tau_{0}}$, also of type $\tau_{0}$, it is completely decomposable by Lemma 86.6 in [3].

The following example illustrates that the homogeneity condition on $H$ in the previous theorem is necessary.

Received by the editors February 5, 1986.

1980 Mathematics Subject Classification (1985 Revision). Primary 20K15, 20K20, $20 \mathrm{~K} 27$. 
EXAMPLE. Let $G=G_{1} \oplus G_{2} \oplus G_{3}$ be a torsion-free group, where $G_{i}$ is of rank one and type $\tau_{i}$ and $G_{i} \not \equiv Q$, the group of rationals, $1 \leq i \leq 3$. Assume that $\tau_{1}$ and $\tau_{2}$ are incomparable and $\tau_{3} \geq \sup \left\{\tau_{1}, \tau_{2}\right\}>\tau_{i}, i=1,2$. By Lemma 1 in [1], $G$ contains an indecomposable pure subgroup $H$ with typeset $T(H)=\left\{\tau_{0}, \tau_{1}, \tau_{2}\right\}$, where $\tau_{0}=\inf \left\{\tau_{1}, \tau_{2}\right\}$. Since $\tau_{i}$ is maximal in $T(H)$,

$$
\left\langle H^{*}\left(\tau_{i}\right)\right\rangle_{*}=\{0\}=H \cap G_{3}=H \cap G^{*}\left(\tau_{i}\right), \quad i=1,2,
$$

and obviously

$$
H=\left\langle H^{*}\left(\tau_{0}\right)\right\rangle_{*}=H \cap G=H \cap G^{*}\left(\tau_{0}\right) .
$$

Thus $H$ is *-pure in $G$.

Proposition 12 in [2] asserts that, if $A$ is a torsion-free almost completely decomposable group, any balanced exact sequence

$$
0 \rightarrow B \rightarrow C \rightarrow A \rightarrow 0
$$

with $C$ completely decomposable is *-balanced. A special case of the following theorem implies that $B$ is also almost completely decomposable.

THEOREM 2. *-pure subgroups of finite rank completely decomposable groups are almost completely decomposable.

PrOOF. Let $H$ be *-pure in a completely decomposable group $G$ and define

$$
\mathcal{E}(H)=\left\{\tau \in T(H):\left\langle H^{*}(\tau)\right\rangle_{*} \varsubsetneqq H(\tau)\right\},
$$

where $T(H)$ is the typeset of $H$. Then, for every $\tau \in \mathcal{E}(H), H(\tau)=H_{\tau} \oplus\left\langle H^{*}(\tau)\right\rangle_{*}$ where $H_{\tau}$ is a nonzero homogeneous completely decomposable group of type $\tau$. If $\tau \in \mathcal{E}(H) \backslash \mathcal{E}(G)$, then $G^{*}(\tau)=G(\tau)$ which implies that $\left\langle H^{*}(\tau)\right\rangle_{*}=H \cap G^{*}(\tau)=$ $H \cap G(\tau)=H(\tau)$, a contradiction. Thus $\mathcal{E}(H) \subseteq \mathcal{E}(G)$ and for every $0 \neq h \in H_{\tau}$, $\tau \in \mathcal{E}(H), \pi_{\tau}(h) \neq 0$.

Let $\tau \in \mathcal{E}(H)$ and let $0 \neq h \in H_{\tau}$. We shall now prove that there exists a homomorphism $\psi: G \rightarrow H_{\tau}$ such that $\psi(h)=n h$ for some nonzero integer $n$. Let $\left\{p_{1}, p_{2}, \ldots, p_{k}\right\}$ be the set of all primes such that

$$
h_{p_{i}}^{G}(h)=h_{p_{i}}^{H}(h) \leq h_{p_{i}}^{G}\left(\pi_{\tau}(h)\right), \quad i=1,2, \ldots, k .
$$

Let $n=\prod_{i=1}^{k} p_{i}^{n(i)}$, where $n(i)=h_{p_{i}}^{G}\left(\pi_{\tau}(h)-h_{p_{i}}^{G}(h)\right), i=1,2, \ldots, k$.

It is easy to see that $n$ divides $\pi_{\tau}(h)$ in $\left\langle\pi_{\tau}(h)\right\rangle_{*}$ and this group is a direct summand of $G_{\tau}=\pi_{\tau}(G)$. We let $\pi_{\tau}(h)=n g^{\prime}$ and let $\theta: G \rightarrow\left\langle\pi_{\tau}(h)\right\rangle_{*}$ be a projection onto $\left\langle\pi_{\tau}(h)\right\rangle_{*}$. Then, for every prime $p \notin\left\{p_{1}, p_{2}, \ldots, p_{k}\right\}$,

$$
h_{p}^{H}(h)=h_{p}^{G}\left(\pi_{\tau}(h)\right)=h_{p}^{G}\left(g^{\prime}\right)
$$

and, for every $p \in\left\{p_{1}, p_{2}, \ldots, p_{k}\right\}$,

$$
h_{p}^{G}\left(\pi_{\tau}(h)\right)=h_{p}^{G}\left(g^{\prime}\right)+h_{p}^{G}\left(\pi_{\tau}(h)\right)-h_{p}^{G}(h)
$$

which implies that $h_{p}^{G}\left(g^{\prime}\right)=h_{p}^{G}(h)$. Thus, the height-sequence of $g^{\prime}$ in $G$ is identical to the height-sequence of $h$ in $G$ which implies that there is an isomorphism $\psi^{\prime}:\left\langle g^{\prime}\right\rangle_{*} \rightarrow\langle h\rangle_{*}$ with $\psi^{\prime}\left(g^{\prime}\right)=h$. We then have

$$
\psi^{\prime} \theta \pi_{\tau}(h)=\psi^{\prime} \theta\left(n g^{\prime}\right)=n \psi^{\prime}\left(g^{\prime}\right)=n h \quad \text { and } \quad \psi=\psi^{\prime} \theta \pi_{\tau} \in \operatorname{Hom}\left(G, H_{\tau}\right) .
$$


We now prove that the regulating subgroup $\sum_{\tau \in \mathcal{E}(H)} H_{\tau}$ of $H$ is completely decomposable. Let $0=\sum_{i=1}^{k} h_{\tau_{i}}$, where $h_{\tau_{i}} \in H_{\tau_{i}}, \tau_{i} \in \mathcal{E}(H), 1 \leq i \leq k$. There exist homomorphisms $\psi_{i}: G \rightarrow H_{\tau_{i}}$ such that $\psi_{i}\left(h_{\tau_{i}}\right)=n_{i} h_{\tau_{i}}$ for some nonzero integers $n_{i}, 1 \leq i \leq k$. If $\tau_{1}$, say, is minimal in $\left\{\tau_{1}, \tau_{2}, \ldots, \tau_{k}\right\}$ we have

$$
0=\sum_{i=1}^{k} \psi_{1}\left(h_{\tau_{i}}\right)=\psi_{1}\left(h_{\tau_{1}}\right)=n_{1} h_{\tau_{1}}
$$

which implies that $h_{\tau_{1}}=0$. It is now easy to see that $h_{\tau_{i}}=0,1 \leq i \leq k$, which implies that $\sum_{\tau \in \mathcal{E}(H)} H_{\tau}=\bigoplus_{\tau \in \mathcal{E}(H)} H_{\tau}$. Thus $H$ is almost completely decomposable.

Recall that a subgroup $B$ of a torsion-free group $A$ is said to be regular in $A$ if, for every $b \in B$, type ${ }_{B}(b)=\operatorname{type}_{A}(b)$. We shall call a subgroup $B$ of a torsion-free group $A$ strongly regular if, for every $b \in B$, there exists a nonzero integer $n$ and a homomorphism $\psi: A \rightarrow B$ such that $\psi(b)=n b$. This definition is an obvious generalization of the concept of strong purity introduced by K. M. Rangaswamy and S. Janakiraman. Strongly regular subgroups are regular but not necessarily pure. A subgroup that is both strongly regular and pure will be called strongly regular pure. Obviously, strongly regular pure subgroups are *-pure but the converse is not true. For finite rank completely decomposable groups we have the following theorem.

THEOREM 3. A subgroup $H$ of a finite rank completely decomposable group $G$ is *-pure if and only if it is strongly regular pure.

ProOF. We need only prove that, if $H$ is *-pure in $G$, then $H$ is strongly regular in $G$. Let $\mathcal{E}(H)$ be defined as in Theorem 2. Then, for $\tau \in \mathcal{E}(H), H(\tau)=$ $H_{\tau} \oplus\left\langle H^{*}(\tau)\right\rangle_{*}$ and, from the proof of Theorem $2, H_{\tau}$ is strongly regular in $G$. We shall prove that $\bigoplus_{\tau \in \mathcal{E}(G)} H_{\tau}$ is strongly regular in $G$.

Let $0 \neq h=\sum_{i=1}^{n} h_{\tau_{i}}$, where $0 \neq h_{\tau_{i}} \in H_{\tau_{i}}, \tau_{i} \in \mathcal{E}(H), 1 \leq i \leq n$. There exist homomorphisms $\psi_{i}: G \rightarrow\left\langle h_{\tau_{i}}\right\rangle_{*}$ and nonzero integers $n_{i}$ such that $\psi_{i}\left(h_{\tau_{i}}\right)=n_{i} h_{\tau_{i}}$, $1 \leq i \leq n$. Then, for every $i \in\{1,2, \ldots, n\}$

$$
\psi_{i}(h)=n_{i} h_{\tau_{i}}+\sum_{j \neq i} \psi_{i}\left(h_{\tau_{j}}\right)
$$

and there exist integers $m_{i}$ and $m_{i}^{\prime}$ such that $m_{i} h_{\tau_{i}}=m_{i}^{\prime}\left(\sum_{j \neq i} \psi_{i}\left(h_{\tau_{j}}\right)\right)$, since $\sum_{j \neq i} \psi_{i}\left(h_{\tau_{j}}\right) \in\left\langle h_{\tau_{i}}\right\rangle_{*}$. Thus

$$
m_{i}^{\prime} \psi_{i}(h)=m_{i}^{\prime} n_{i} h_{\tau_{i}}+m_{i}^{\prime}\left(\sum_{j \neq i} \psi_{i}\left(h_{\tau_{j}}\right)\right)=r_{i} h_{\tau_{i}}, \quad \text { where } r_{i}=m_{i}^{\prime} n_{i}+m_{i}
$$


Thus

$$
\begin{aligned}
\left(\prod_{i=1}^{n} r_{i}\right) h & =\sum_{i=1}^{n}\left(\prod_{i=1}^{n} r_{i}\right) h_{\tau_{i}}=\sum_{i=1}^{n}\left(\prod_{j \neq i} r_{j}\right) r_{i} h_{\tau_{i}} \\
& =\sum_{i=1}^{n} r_{i}^{\prime} m_{i}^{\prime} \psi_{i}(h), \quad \text { where } r_{i}^{\prime}=\prod_{j \neq i} r_{j} \\
& =\left(\sum_{i=1}^{n} r_{i}^{\prime} m_{i}^{\prime} \psi_{i}\right)(h) .
\end{aligned}
$$

Hence

$$
\psi=\sum_{i=1}^{n} r_{i}^{\prime} m_{i}^{\prime} \psi_{i} \in \operatorname{Hom}\left(G, \bigoplus_{\tau \in \mathcal{E}(H)} H_{\tau}\right)
$$

and $\psi(h)=r h$, where $r=\prod_{i=1}^{n} r_{i}$. This implies that $\bigoplus_{\tau \in \mathcal{E}(H)} H_{\tau}$ is strongly regular in $G$. Since $H / \bigoplus_{\tau \in \mathcal{E}(H)} H_{\tau}$ is finite, for every $h^{\prime} \in H$ there exists an integer $n^{\prime}$ such that $n^{\prime} h^{\prime} \in \bigoplus_{\tau \in \mathcal{E}(H)} H_{\tau}$ and from the previous paragraphs there exists $\psi: G \rightarrow \bigoplus_{\tau \in \mathcal{E}(H)} H_{\tau} \subseteq H$ such that $\psi\left(n^{\prime} h^{\prime}\right)=r n^{\prime} h^{\prime}=n^{\prime} \psi\left(h^{\prime}\right)$ for some nonzero integer $r$. By torsion-freeness, $\psi\left(h^{\prime}\right)=r h^{\prime}$ which implies that $H$ is strongly regular in $G$ and the proof is complete.

From the proof of the previous theorem we conclude that

(i) finite rank *-pure subgroups of separable torsion-free groups are strongly regular,

(ii) completely decomposable *-pure subgroups of completely decomposable groups are strongly regular.

It seems likely that the concepts of $*$-purity and strongly regular purity are equivalent for separable torsion-free groups.

Finally, we wish to characterize those completely decomposable groups whose typesets satisfy the maximum condition any *-pure subgroup of which is completely decomposable.

A set of types $\tau$ is called a tree if, for any two incomparable types $\tau_{1}$ and $\tau_{2}$ in $\tau$, there is no $\tau$ in $\tau$ satisfying $\tau \geq \sup \left\{\tau_{1}, \tau_{2}\right\}$.

THEOREM 4. Let $G$ be a reduced completely decomposable group whose typeset satisfies the maximum condition. Every *-pure subgroup of $G$ is completely decomposable if and only if $\mathcal{E}(G)$ is a tree.

PROOF. Suppose every *-pure subgroup of $G$ is completely decomposable. Let $\tau_{1}, \tau_{2} \in \mathcal{E}(G)$ be incomparable. If there exists $\tau \in \mathcal{E}(G)$ such that $\tau \geqslant \tau_{i}, i=1,2$, then by the example after Theorem $1, G$ contains a *-pure subgroup which is not completely decomposable, a contradiction. This implies that $\mathcal{E}(G)$ is a tree.

Conversely, assume that $\mathcal{E}(G)$ is a tree and let $H$ be a *-pure subgroup of $G$. Define

$$
\mathcal{E}(H)=\left\{\tau \in T(H):\left\langle H^{*}(\tau)\right\rangle_{*} \varsubsetneqq H(\tau)\right\}
$$


Then $\mathcal{E}(H)$ is nonempty since every maximal element of $T(H)$ belongs to $\mathcal{E}(H)$. For every $\tau \in \mathcal{E}(H)$

$$
\begin{aligned}
H(\tau) /\left\langle H^{*}(\tau)\right\rangle_{*} & =(H \cap G(\tau)) /\left(H \cap G^{*}(\tau)\right) \\
& \cong\left\{(H \cap G(\tau))+G^{*}(\tau)\right\} / G^{*}(\tau) \\
& \subseteq G(\tau) / G^{*}(\tau) \cong G_{\tau} .
\end{aligned}
$$

Thus $H(\tau) /\left\langle H^{*}(\tau)\right\rangle_{*}$ is a homogeneous group of type $\tau$ isomorphic to a subgroup of $G_{\tau}$ and therefore completely decomposable. This also implies that $\left\langle H^{*}(\tau)\right\rangle_{*}$ is balanced in $H(\tau)$ and therefore $H(\tau)=H_{\tau} \oplus\left\langle H^{*}(\tau)\right\rangle_{*}$ where $H_{\tau}$ is a homogeneous completely decomposable group of type $\tau$. From the proof of Theorem $2, H_{\tau}$ is strongly regular in $G$ for every $\tau \in \mathcal{E}(H)$ and $\sum_{\tau \in \mathcal{E}(H)} H_{\tau}=\bigoplus_{\tau \in \mathcal{E}(H)} H_{\tau}$, a completely decomposable group. We shall prove that $H=\bigoplus_{\tau \in \mathcal{E}(H)} H_{\tau}$.

Let $h \in H$ be of type $\tau_{0}$ and, since $T(H)$ satisfies the maximum condition, we may assume that every element of $H$ of type greater than $\tau_{0}$ belongs to $\bigoplus_{\tau \in \mathcal{E}(H)} H_{\tau}$, i.e. $H^{*}\left(\tau_{0}\right) \subseteq \bigoplus_{\tau \in \mathcal{E}(H)} H_{\tau}$. We first show that this assumption implies that $\left\langle H^{*}\left(\tau_{0}\right)\right\rangle_{*} \subseteq \bigoplus_{\tau \in \mathcal{E}(H)} H_{\tau}$. Let $h^{\prime} \in\left\langle H^{*}\left(\tau_{0}\right)\right\rangle_{*}$ and let

$$
\mathcal{E}\left(h^{\prime}\right)=\left\{\tau \in \mathcal{E}(G): \pi_{\tau}(h) \neq 0\right\} .
$$

Since $\left\langle H^{*}\left(\tau_{0}\right)\right\rangle_{*}=H \cap G^{*}\left(\tau_{0}\right), \tau \geqslant \tau_{0}$ for every $\tau \in \mathcal{E}\left(h^{\prime}\right)$. Let $\mathcal{M}$ be the set of all minimal types in $\mathcal{E}\left(h^{\prime}\right)$ and, for every $\tau \in \mathcal{M}$, let

$$
\mathcal{M}(\tau)=\left\{\tau^{\prime} \in \mathcal{E}\left(h^{\prime}\right): \tau^{\prime} \geq \tau\right\} .
$$

Since $\mathcal{E}(G)$ is a tree, $\{\mathcal{M}(\tau): \tau \in \mathcal{M}\}$ is a partition of $\mathcal{E}\left(h^{\prime}\right)$ and also $\sum_{\tau \in \mathcal{M}} G(\tau)=$ $\bigoplus_{\tau \in \mathcal{M}} G(\tau)$, a summand of $G$. From the proof of Theorem $3 \bigoplus_{\tau \in \mathcal{E}(H)} H_{\tau}$ is strongly regular in $G$ and, by assumption, there exists a nonzero integer $n$ such that $n h^{\prime} \in \bigoplus_{\tau \in \mathcal{E}(H)} H_{\tau}$. Thus, there is a homomorphism $\psi: G \rightarrow \bigoplus_{\tau \in \mathcal{E}(H)} H_{\tau}$ such that $\psi\left(h^{\prime}\right)=k h^{\prime}$ for some nonzero integer $k$. Then

$$
k h^{\prime}=\sum_{\tau \in \mathcal{E}\left(h^{\prime}\right)} \psi \pi_{\tau}\left(h^{\prime}\right),
$$

where $\tau_{0} \leq \tau \leq$ type $_{H}\left(\psi \pi_{\tau}\left(h^{\prime}\right)\right)$ for every $\tau \in \mathcal{E}\left(h^{\prime}\right)$. By assumption, this implies that $\psi \pi_{\tau}\left(h^{\prime}\right) \in \bigoplus_{\tau \in \mathcal{E}(H)} H_{\tau}$. We then have

$$
\begin{aligned}
k h^{\prime} & =\sum_{\tau \in \mathcal{E}\left(h^{\prime}\right)} \psi \pi_{\tau}\left(h^{\prime}\right)=\sum_{\tau \in \mathcal{M}} \sum_{\tau^{\prime} \in \mathcal{M}(\tau)} \psi \pi_{\tau^{\prime}}\left(h^{\prime}\right) \\
& \in \sum_{\tau \in \mathcal{M}}\left\langle\psi \pi_{\tau^{\prime}}\left(h^{\prime}\right): \tau^{\prime} \in \mathcal{M}(\tau)\right\rangle_{*},
\end{aligned}
$$

where $\left\langle\psi \pi_{\tau^{\prime}}\left(h^{\prime}\right): \tau^{\prime} \in \mathcal{M}(\tau)\right\rangle_{*}$ is a pure subgroup of $G$ for every $\tau \in \mathcal{M}$. Thus

$$
\sum_{\tau \in \mathcal{M}}\left\langle\psi \pi_{\tau^{\prime}}\left(h^{\prime}\right): \tau^{\prime} \in \mathcal{M}(\tau)\right\rangle_{*}=\bigoplus_{\tau \in \mathcal{M}}\left\langle\psi \pi_{\tau^{\prime}}\left(h^{\prime}\right): \tau^{\prime} \in \mathcal{M}(\tau)\right\rangle_{*}
$$

is pure in $\bigoplus_{\tau \in \mathcal{M}} G(\tau)$ and therefore pure in $G$. This implies that

$$
h^{\prime} \in \bigoplus_{\tau \in \mathcal{M}}\left\langle\psi \pi_{\tau^{\prime}}\left(h^{\prime}\right): \tau^{\prime} \in \mathcal{M}(\tau)\right\rangle_{*} \subseteq \bigoplus_{\tau \in \mathcal{E}(H)} H_{\tau}
$$


which proves that $\left\langle H^{*}\left(\tau_{0}\right)\right\rangle_{*} \subseteq \bigoplus_{\tau \in \mathcal{E}(H)} H_{\tau}$. But then $h \in H\left(\tau_{0}\right)=H_{\tau} \oplus\left\langle H^{*}\left(\tau_{0}\right)\right\rangle_{*}$ which proves that $H=\bigoplus_{\tau \in \mathcal{E}(H)} H_{\tau}$ is completely decomposable.

In the general case, one observes that if $\mathcal{E}(G)$ (and therefore $T(G)$ ) is linearly ordered, then every pure subgroup of $G$ is *-pure. One can therefore characterize completely decomposable groups any *-pure subgroup of which is completely decomposable by combining Theorem 2 in [1] and Theorem 4 above.

\section{REFERENCES}

1. L. Bican, Completely decomposable abelian groups any pure subgroup of which is completely decomposable, Czechoslovak Math. J. 24 (1974), 176-191.

2. H. Bowman and K. M. Rangaswamy, On special balanced subgroups of torsion-free abelian groups, preprint.

3. L. Fuchs, Infinite abelian groups, Vol. 2, Academic Press, New York, 1973.

4. Loyiso G. Nongxa, Homogeneous subgroups of completely decomposable groups, Arch. Math. 42 (1984), 208-213.

Department of Mathematics and Applied Mathematics, University of Natal, King George V Avenue, Durban 4001, South Africa 